\title{
Surveillance of Clostridium difficile infections in a long-term care psychogeriatric facility: outbreak analysis and policy improvement
}

\author{
Gretel Van Esch', Johan Van Broeck ${ }^{2}$, Michel Delmée ${ }^{2}$ and Boudewijn Catry ${ }^{3 *}$
}

\begin{abstract}
Background: Following an exceptionally high Clostridium difficile infections (CDI) incidence (Spring 2011) in a psychogeriatric long-term care facility, a bidirectional study (2009-2012) was initiated to identify determinants (retrospectively) and to assess intervention measures taken (prospectively).
\end{abstract}

Methods: For every CDI patient (de novo cases, relapses, and recurrences), a control patient (patient in the opposite room) was selected and risk factor analysis performed. Following the epidemic peak a more stringent hygienic protocol and surveillance program were implemented, as well as uniform guidelines for metronidazole and vancomycin prescription.

Results: The nutritional state (total protein/prealbumine) significantly differed between the CDI group (poorer nutritional state at admission) and the control group, and also antibiotic use (general) could be confirmed as a risk factor. A multi-disciplinary nutritional team has been established in order to improve the nutritional balance of our patients.

Conclusions: Aside from stringent hygiene and antibiotic prescription stewardship, malnutrition of patients is a factor to be taken into account to contain a CDI outbreak in a long term care facility (LTCF).

Keywords: Clostridium difficile, Intervention, Nutritional status, LTCF

\section{Background}

Clostridium difficile is an anaerobic Gram-positive, sporeforming bacterium [1]. The ability of $C$. difficile to form spores is thought to be a key feature in enabling the bacteria to persist in patients and in the physical environment for long periods, thereby facilitating its transmission. $C$. difficile is transmitted through the fecal-oral route. The risk factors for acquiring $C$. difficile include contact with a contaminated environment, contact with persons who are infected with and shedding $C$. difficile, and ingestion of contaminated food [2].

As the many spores formed by $C$. difficile are resistant to most routine cleaning methods used on surfaces in hospitals (except for diluted bleach), $C$. difficile infection (CDI) has become the leading cause of diarrhea and

\footnotetext{
* Correspondence: Boudewijn.Catry@wiv-isp.be

${ }^{3}$ Healthcare-associated Infections (NSIH) \& Antimicrobial Resistance, OD Public Health \& Surveillance, Scientific Institute of Public Health, Brussels (WIV-ISP), Rue Juliette Wytsmanstraat 14, 1050 Brussels, Belgium

Full list of author information is available at the end of the article
}

pseudomembranous colitis in healthcare settings [3]. There is a strong connection between antimicrobial therapy and $\mathrm{CDI}$, as $C$. difficile can only colonize the gut if the normal intestinal flora is disturbed or absent [3]. In recent years, an increase in the incidence and the severity of healthcareassociated CDI (nosocomial) has been reported worldwide. This had been attributed to multiple factors including a changing demographic situation, increased use of broadspectrum antibiotics and the emergence of hypervirulent C. difficile strains. Outbreaks were associated with the emergence and rapid spread of a specific strain of $C$. difficile belonging to the PCR-ribotype 027 or pulsotype NAP1 (North American Pulsotype 1) [3]. Some of the characteristics of this strain are higher in vitro production of toxins $\mathrm{A}$ and $\mathrm{B}$ and presence of a third toxin named binary toxin. Since about 2005, the epidemic strain has started to circulate in northern Europe (United Kingdom, Belgium and the Netherlands) [1]. 
Older people are particularly susceptible and at increased risk for adverse outcomes as a result of CDI. The increased risk of acquiring CDI in the elderly may be due to age-related changes in intestinal flora, immune senescence, or the presence of other underlying diseases [3].

In Belgium, CDI incidence more than doubled between 1998 and 2007, after which it stabilized, be it at a high level [4,5]. After an initial increase, the CDI mortality has been decreasing since 2004 [4]. PCR ribotype 027 was identified for the first time in 2003. Although strains attributed to severe pathology are generally found in hospital patients, recent reports suggest that the occurrence and severity of CDI in the community is also increasing. Moreover, CDI is more and more recognized as a cause of diarrhea in populations previously considered to be at low risk. The carriage ratio of $C$. difficile in the communitydwelling elderly population in the UK is 4\% [6]. The patient population in the psychogeriatric institutions is marked by cognitive impairment which hampers an optimal maintenance of standards of personal hygiene. Patients are often unconscious of their hygienic condition and nursing staff regularly has to deal with noncooperative behavior regarding personal hygiene.

The long-term psychogeriatric facility, from which the data in this manuscript are observed, had an increase of CDI between 2009-2010 and 2012. However in the beginning of 2011, a substantial increase of CDI episodes was recorded in the institute. The objective of the manuscript was to investigate underlying causes of this epidemic episode in order to identify the risk factors within this specific population and health care facility.

\section{Methods}

\section{Study designs}

\section{A. Retrospective design}

To identify risk factors for CDI, data covering the period of January 2009 to December 2012 were retrospectively abstracted from the patient's record in four departments of one psychogeriatric facility. The hospital had a total capacity of 120 beds and the mean length of stay was 34 days. The effect was evaluated statistically.

\section{B. Prospective design}

To improve the CDI policy within institutions, an active surveillance and prevention campaign (= intervention) was launched during the epidemic peak in Spring 2011, including hygiene precautions for health care professionals and visitors. The effect was evaluated descriptively until the end of the study period, namely December 2012.

\section{Intervention}

A more stringent hygienic protocol and surveillance program were implemented in order to better battle the current outbreak and to sooner detect potential future outbreaks: All CDI occurrences were reported back to a single reference person and analyzed on a three-monthly basis. When an increase by more than two standard deviations above the normal background level was observed, the following measures were taken: (a) staff was alerted of the increased CDI incidence and the importance of the existing hygienic measures was explicitly impressed upon all personnel and visitors; (b) strict isolation measures were immediately taken for patients with diarrhea awaiting the laboratory results on their fecal samples; (c) patients with high suspicion for CDI (characteristic odor and mucal composition of diarrhea) were immediately treated with metronidazole (p.o. $3 \times 500 \mathrm{mg}$ daily for 10 days) or vancomycin (p.o. $4 \times 125 \mathrm{mg}$ daily for 10 days); (d) patients with confirmed CDI must have been treated with vancomycin and were kept in isolation during the full course of their CDI treatment (10 days for de novo cases, 21 for relapses); (e) at the end of any CDI treatment, patient rooms were cleaned with diluted bleach $(\mathrm{NaClO})$ prior to the standard disinfection procedure. This cleaning procedure was once more repeated when the concerned patient was discharged.

A request for ribotyping was submitted to the local ethical committee (Number of acceptance: 2012/03/ $09 / 2$ ) in order to assess which strain was circulating within the institution.

Upon approval (29 May 2012), a ribotyping was done among all consecutive patients within the span of one year (10 patients in total) in the aftermath of the 2011 peak.

\section{Case definition \& bacteriology}

A Clostridium difficile infection (CDI) was defined if a patient showed diarrhea as the major clinical symptom and had a positive toxin/antigen test (C. diff quik chek complete $^{\circ}$ - Alere Techlab) and a positive stool culture.

Cases were classified as (I) de novo case: no prior infection known; (II) relapse: CDI within 2 weeks after completing the treatment, and (III) late relapse (or recurrence): CDI later than 2 weeks after completing the treatment.

C. difficile culture was performed according to local standard procedures (CPG laboratory Brussels) (selective media: CLO medium, incubation at $35^{\circ}$ for 48 hours under anaerobic conditions). Colonies were identified based on Gram-staining, typical odor and chartreuse fluorescence under ultraviolet light.

C. difficile isolates (only from the peak of 2011 onwards, i.e. after initiation of intervention), were sent to the national reference laboratory [7] (UCL Brussels) for typing. Strains were characterized by PCR-ribotyping, based on the comparison of patterns of PCR products of the 16S-23S rRNA intergenic spacer regions using primers described by Barbut et al. [8]. The size of each peak was determined using GeneScan software or GeneMapper V.4 software (AB - Applied Biosystems) [5]. 


\section{Patient data recovery and analysis}

For all CDI episodes, patients' files were reviewed (G VE). For each patient, the patient in the opposite room served as control. The parameters that were extracted are presented in Table 1.

The statistical analysis of the patient parameters was performed based on the CDI incidence. This approach inherently applied the appropriate weight factor to the patient parameters of the more susceptible patients, as the data for a patient who had two infections were counted twice. All statistical analysis were performed either with Microsoft Excel 2007 or with SPSS Statistics version 17.0. (SPSS Inc, Chicago, IL). The $t$-test for independence and Chi squared-test for nominal data were applied, with significance level set at 0,05 .

\section{Table 1 Overview of patient characteristics abstracted from the medical records of Clostridium difficile cases and controls in a long-term psychogeriatric health care institute, Belgium 2011}

General patient characteristics:

Age

Body Mass Index (BMI)

Frailty index (FI)

Nutritional status

Mini Mental State Examination (MMSE)

CDI specific characteristics:

Interval between admission and infection

Length of stay

Type of treatment for CDI

Length of $\mathrm{CDI}$ treatment

Mortality

(years) at admission

$\left(\mathrm{kg} / \mathrm{m}^{2}\right)$ at admission

at admission and at discharge according to Drubbel et al. [9] (scaling between 1 and 0,0 being perfectly healthy)

(mg/dL or $\mathrm{g} / \mathrm{L}$ ) at admission based on prealbumine: $(<20 \mathrm{mg} / \mathrm{dl}=$ malnutrition, $>20 \mathrm{mg} / \mathrm{dL}=$ normal). If not available, based on total protein: $<5,0 \mathrm{~g} / \mathrm{L}$ malnutrition, $>5,0 \mathrm{~g} / \mathrm{L}$ normal) [10]

$(x / 30)$ during stay to assess the cognitive functioning. MMSE below 21/30 is a usual cut-off to define dementia [11]

(days), the date at which lab results confirm the clinical diagnosis is taken as the date of infection

(days) between admission and discharge

metronidazole or vancomycin

(days)

(\%)

Literature reported CDI risk factors:

Type of residency

one month prior to admission, i.e. previous health care exposure (acute hospital, nursing home, LTCF)

Number and type of antibiotics

used one month prior to CDI

Antacids

used at admission (proton pump inhibitor or histamin-2 blockers or none)

Number of narcotics

used at admission
Diabetes mellitus type 2

at admission (yes/no)

CDI: Clostridium difficile infection; LTCF: long-term care facility.

\section{Results}

\section{A. Retrospective patient study}

The study population consisted of 66 patients affected by $C$. difficile and 61 control patients. A total of 94 episodes were included in the study: 42 patients had one single episode of CDI during their hospitalization in the institution and 24 patients relapsed or had a late relapse. Among the latter, 20 patients had 2 episodes (16 relapses and 4 late relapses), 3 patients with 3 episodes (4 relapses and 2 late relapses), and 1 patient with 4 episodes of CDI (3 relapses and 0 late relapses), respectively.

A baseline CDI incidence of 16 cases/1000 admissions was found for the years 2009, 2010, and 2012, respectively. Figure 1 shows the epidemic increase in the number of CDI cases in 2011. The increase included the total number of CDI episodes, i.e. de novo cases, relapses and late relapses.

The institution underwent an increased CDI incidence as also reported by others during the same time frame in Europe [12]. Once introduced, the highly infectious strain spreads in-house: $42 \%$ of the relapses occurred for patients in rooms previously occupied by an infected patient or in adjacent rooms.

\section{General patient characteristics}

Table 2 gives the association comparing patients' characteristics of any CDI events and controls. The analysis accounted for the fact that patients with a relapse would have been counted more than once. Patients in the infected group showed near-identical general characteristics compared to patients in the control group. No difference was found regarding their mean age, body mass index (BMI), frailty index or MMSE (Mini Mental State Examination).

\section{CDI specific characteristics}

As discussed in the above section, especially during the 2011 CDI incidence peak, the institute had an important influx of patients carrying the CDI. Of all positive cultures, $18 \%$ were obtained within one week after admission and up to $25 \%$ within two weeks. We can assume that the majority of the former had contracted $C$. difficile before admission in our hospital.

The mean length of stay for the control group was 55 days and for the CDI patient group 85 days. Logically, the infection prolonged their mean length of stay, partially due to the length of the CDI treatment itself, and partially due to their weakened state thereafter requiring additional care.

Figure 2 presents the antimicrobial treatment in function of case classification. The odds of relapse were 4,3 times (95\% CI: 0,867 - 21,550) higher in patients treated with metronidazole treatment compared to vancomycin treatment. Metronidazole reduced sensitivity is known 


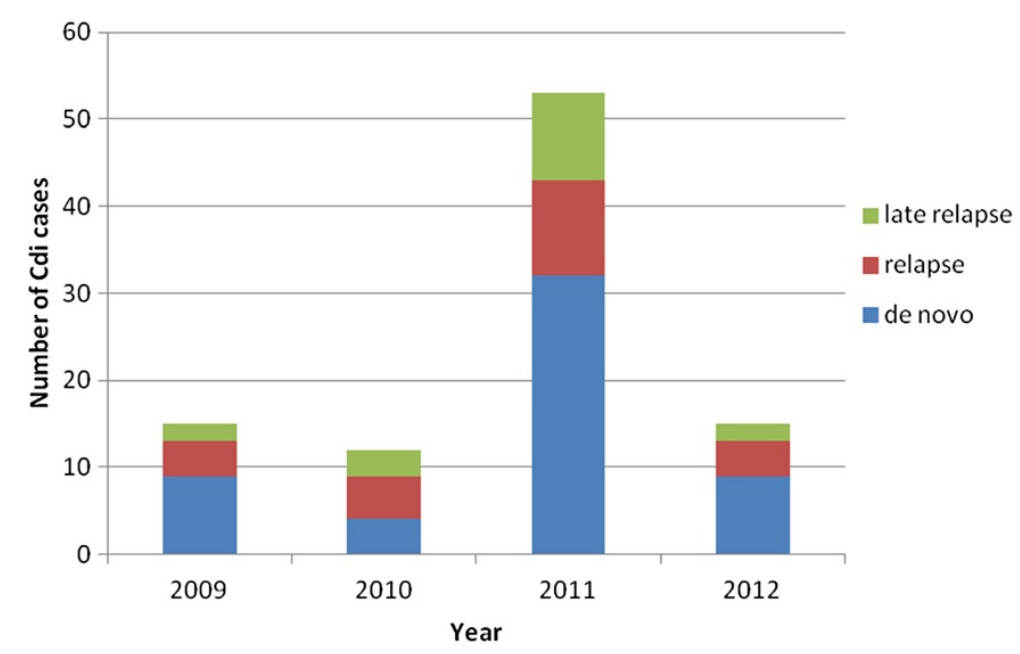

Figure $1 \mathrm{Graphical}$ overview of the incidence of Clostridium difficile infection (CDI) in a long-term psychogeriatric institution between 2009 and 2012, Belgium.

for the ribotype 027 strain [13]. Although no ribotyping was performed at the moment of the outbreak, this finding suggested that we dealt with the same strain as the one previously identified in the acute hospital setting. The odds for relapse when comparing a short versus longer treatment, i.e. less than 14 days or more than 14 days, showed no benefit for the longer treatment in our study population (odds ratio $=0,9(95 \%$ CI: 0,300 - 2,502)).

The mortality in the CDI group was $21 \%$ compared to $10 \%$ in the control group.

\section{Literature reported CDI risk factors}

Since literature reported CDI risk factors, as selected for this study, have all been evaluated at admission, we have chosen to perform this statistical analysis based on the data per patient rather than per CDI incidence event. An overview of the statistical analysis is shown in Table 3. From the risk factors previously discussed in literature, only antibiotic use differs significantly between the control group and the CDI patients in our study population.

\section{B. Prospective CDI policy improvement}

Although hygienic procedures were available at the moment of the outbreak, raising awareness and improved internal communication were the first steps to be undertaken. Figure 3 shows that the implementation of the infection control measures (May-June 2011) coincided with a significant drop in CDI incidence. Although the epidemiological characteristics combined with the metronidazole reduced sensitivity strongly point towards infections with the 027 strain, ribotyping was not able to confirm this suspicion, given the time delay between the outbreak and the approval from the ethical committee.

Upon approval of the request (May 2012), all consecutive patients $(n=10)$ within the time frame of one year, were ribotyped, but no 027 strain was confirmed in any of these samples. Reassuringly, these findings substantiate the effectiveness of the more stringent hygienic protocol for battling an infectious outbreak.

The patient parameter study has led to two additional measures; (a) guidelines for metronidazole and vancomycin

Table 2 Comparison of patients' characteristics of Clostridium difficile infection (CDI) events and controls

\begin{tabular}{|c|c|c|c|c|c|c|c|}
\hline & \multicolumn{3}{|c|}{ Controls } & \multicolumn{3}{|c|}{ CDI infections } & \multirow[t]{2}{*}{ p-value ${ }^{\S}$} \\
\hline & Mean & St. Dev. & Range & Mean & St. Dev. & Range & \\
\hline Age (years) & 82 & 7,3 & (64-99) & 82 & 7 & $(66-97)$ & 0,77 \\
\hline $\mathrm{BMI}\left(\mathrm{kg} / \mathrm{m}^{2}\right)$ & 23 & 5,1 & $(15-37)$ & 23 & 5,5 & $(15-40)$ & 0,88 \\
\hline Fl-admission & 0,38 & 0,09 & $(0,25-0,55)$ & 0,38 & 0,07 & $(0,22-0,53)$ & 0,62 \\
\hline total protein $(\mathrm{g} / \mathrm{L})$ & 5,8 & 0,9 & $(4-8)$ & 5,4 & 0,8 & $(4-8)$ & $0,03^{*}$ \\
\hline Prealbumine (mg/dL) & 20,6 & 7 & $(8-35)$ & 16,6 & 7,4 & $(5-41)$ & $0,03^{*}$ \\
\hline MMSE (x/30) & 22 & 6 & $(7-30)$ & 21 & 7,3 & $(0-30)$ & 0,68 \\
\hline
\end{tabular}

${ }_{\mathrm{s}} \mathrm{p}$-value: from independent $t$-test; ${ }^{*}$ : significant at $\mathrm{p}<0,05$. For abbreviations: see Table 1. 


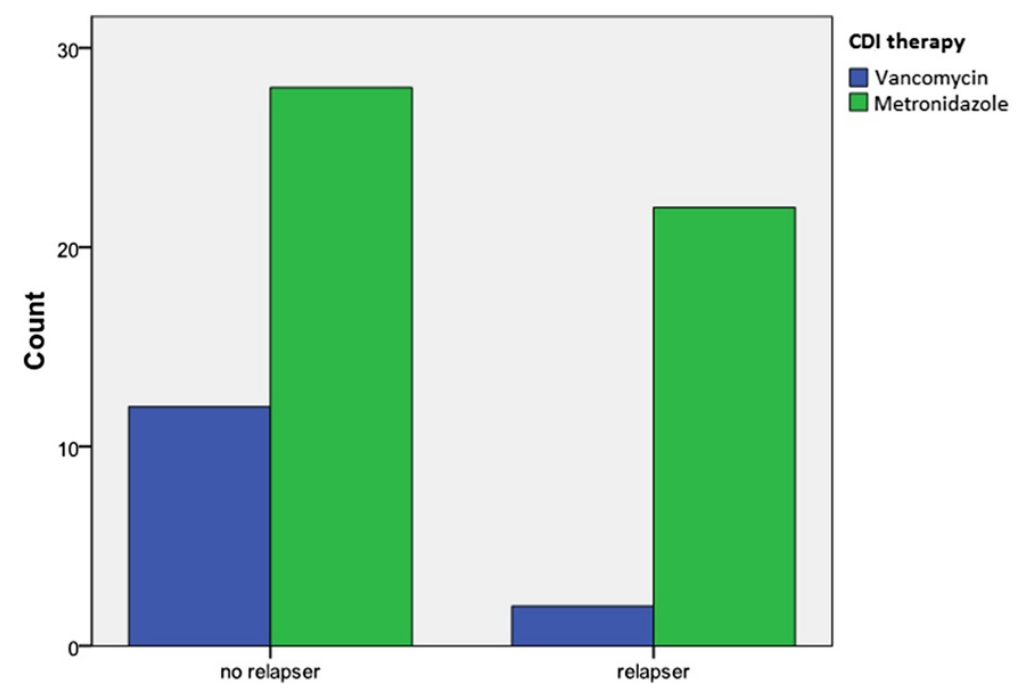

Figure 2 Vancomycin (blue) or metronidazole (green) treatment counts for Clostridium difficile infections (CDI), separated between relapse and non-relapse cases.

prescription have now been implemented as described above, and (b) if malnutrition appears to be an underlying problem and a possible additional risk-factor, a multidisciplinary nutritional team, consisting of a psychologist, dietician and physiotherapist is established in order to improve the nutritional balance of the patients.

\section{Discussion}

Only the nutritional status, based on the total protein and prealbumine levels, was found to be significantly poorer for the patients that suffered CDI: whereas patients in the control group on average were classified as normal (mean prealbumine $>20 \mathrm{mg} / \mathrm{dL}$ ), patients in the infected group already suffered from malnutrition at admission (mean prealbumine $<20 \mathrm{mg} / \mathrm{dL}$ ). Their total protein level was also significantly lower. A multi-disciplinary nutritional team thus can be considered in long term care facilities to further contain $C$. difficile outbreaks.

Within the general patient characteristics, the frailty index was higher than reported by Drubbel et al. [9]. They reported a median FI score of 0,08 for women and 0,06 for men. Our median FI was 0,39. A slight discrepancy could arise from the fact that we calculated the FI manually, based on the patients files and not automatically as done by Drubbel and her group. However, the main difference is related to the patient population itself: in contrast to their study population (community-dwelling elderly people), here we only focused on inpatients.

To our knowledge, there is only one study evaluating cognitive impairment in relation with CDI. Kyne et al. [14] reported in 1999 that cognitive impairment is a risk factor for severity of CDI. Although here, we also hypothesized that cognitive impairment might be an additional risk factor for contracting or developing CDI, our data did not support this hypothesis: no difference was observed between the MMSE data of both groups. Given the high frailty index (FI) in the population of our study, it should be noted that both groups are dependent on the same external help for their hygienic situation, independent of their cognitive state. Thirty-eight percent of our C. difficile patients and $36 \%$ of our control patients went to a nursing home after discharge.

Regarding the CDI specific parameters, we noticed an overall mortality rate of $21 \%$ in our CDI group. This number is in the range of other mortality rates published before $[15,16]$. As we did not calculate the attributable mortality of CDI, we probably overestimate the CDI mortality rate because we did not correct for other factors like high age and multiple underlying diseases [17].

The increasing incidence and severity of CDI is largely described in literature [12]. Viseur et al. published the surveillance data for Belgium from 2008 to 2010 [5]. Data from these years showed a relatively stable incidence of CDI in Belgian hospitals, with a slight decrease in 2010. Surveillance data until 2011 are available on the website of the Public Health and Surveillance Department, Scientific Institute for Public Health (WIV-ISP), Brussels [4]. The overall incidence of CDI in 2011 is comparable with 2010, but between 2007 and 2011, there were more outbreaks of CDI in long-term care hospitals than in the acute hospital setting (60\% vs. $38 \%)$. Our data fits within this trend. The background incidence (16 CDI per 1000 admission) is comparable to the data reported by Mascart et al. in a geriatric department (Centre Hospitalier Universitaire Brugmann, Brussels), located in the same geographic area 
Table 3 Statistical analysis of the literature reported Clostridium difficile infection (CDI) risk factors for all patients in the study

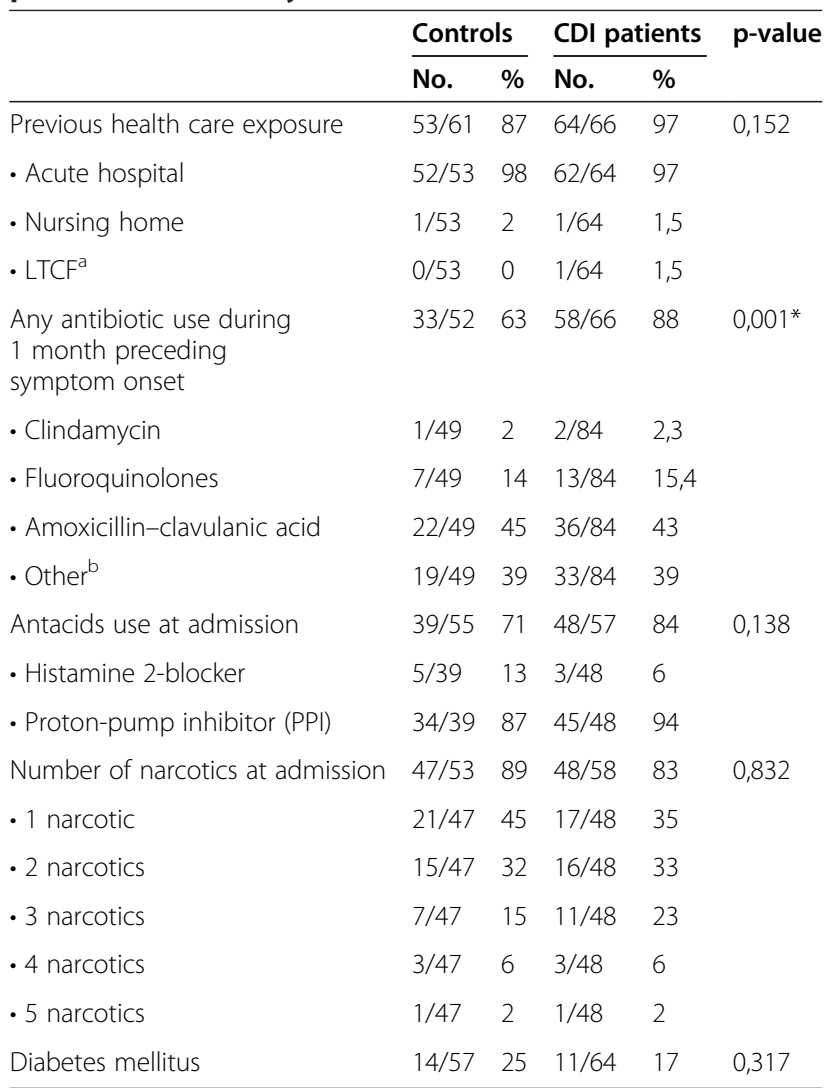

aTCF: long-term care facility.

b'other' includes cephalosporins, tetracyclines, macrolides and cotrimoxazole (trimethoprim + sulphonamides).

The last column reports the $\mathrm{p}$-value of $\mathrm{Chi}^{2}$-test for nominal data. The values marked with the asterix are significant at the 0,05 level.
[15]. They observed an incidence of 13/1000 admissions in 2008 and $21 / 1000$ admissions in 2009. The ribotype 027 was the most prevalent ribotype during the years 2007-2009. Unfortunately no recent data are available to support the hypothesis of the ribotype 027 involvement in outbreaks among patients in geriatric wards.

Based upon experience, vancomycin could be recommended as the first-line agent to protect against recurrence and control of a hospital outbreak caused by a hypervirulent strain. Control is difficult but possible through a combination of barrier precautions, environmental cleaning, and antimicrobial stewardship, interventions that all are concordant with literature $[13,15]$.

\section{Conclusions}

In summary, following the 2011 CDI outbreak in the institution in which the study was conducted a more stringent hygienic protocol and surveillance program were implemented in order to sooner detect potential outbreaks. Additionally, uniform guidelines for metronidazole and vancomycin prescription have been implemented. Finally, a multi-disciplinary nutritional team has been established in order to improve the nutritional balance of our patients.

\section{Ethical standards}

The authors assert that all procedures contributing to this work comply with the ethical standards of the relevant national and institutional committees on human experimentation and with the Helsinki Declaration of 1975, as revised in 2008 .

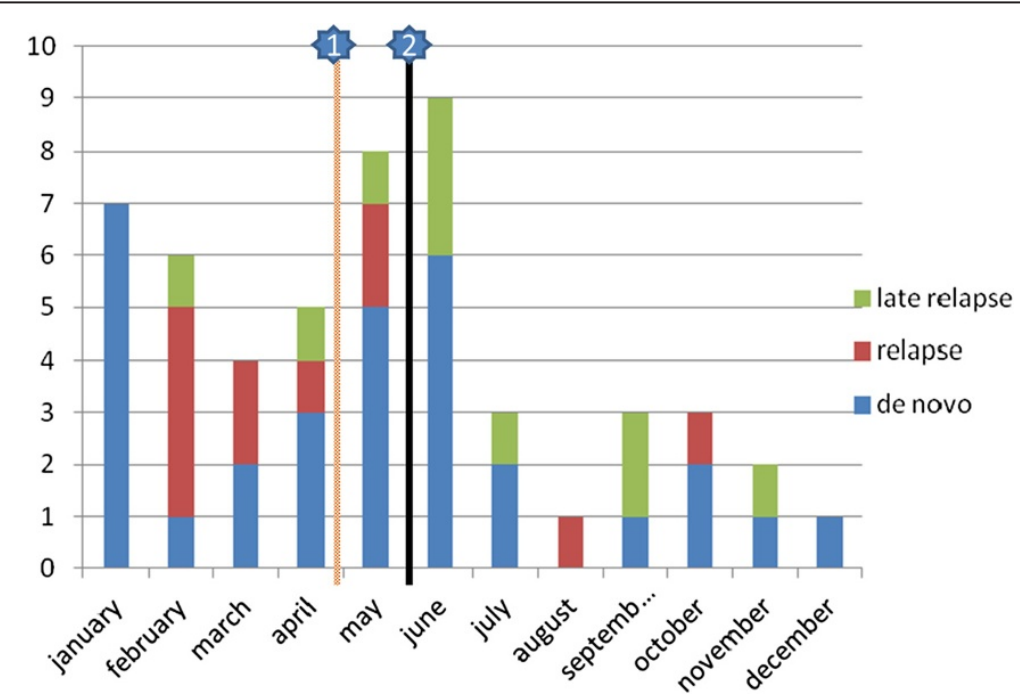

Figure 3 Graphical overview of the monthly incidence of Clostridium difficile infection (CDI) in the institution in 2011. Line 1: First awareness moment with communication of increase in incidence. Line 2: Formal intervention start, with stringent hygienic protocol, surveillance and uniform antibiotic guidelines. 


\section{Competing interests}

The authors declare that they have no competing interests.

\section{Authors' contributions}

GVE conceived of the study, carried out the data collection, analysis and drafted the manuscript. JVB and MD give guidance on microbiology, performed the ribotyping and revised the manuscript. BC helped in the study design, interpretation of the data and drafting of the manuscript. All authors read and approved the final manuscript.

\section{Financial support}

The C. difficile cultures were performed by the CPG laboratory (Herbert Diquas, Colette Jouniaux et al.), Brussels, Belgium. PCR Ribotyping was performed by the national reference laboratory for Clostridium difficile, UCL, Brussels.

We thank Professor Dr Herman Van Oyen for his support during the conception, design, review and interpretation of the analysis, and revision of the manuscript.

\section{Author details}

${ }^{1}$ Scheutbos, Revalidation Centre for Psychogeriatric patients, Brussels, Belgium. ${ }^{2}$ Department of Clinical Microbiology, Cliniques Universitaires Saint-Luc (UCL), Brussels, Belgium. ${ }^{3}$ Healthcare-associated Infections (NSIH) \& Antimicrobial Resistance, OD Public Health \& Surveillance, Scientific Institute of Public Health, Brussels (WIV-ISP), Rue Juliette Wytsmanstraat 14, 1050 Brussels, Belgium.

Received: 1 December 2014 Accepted: 19 February 2015

\section{Published online: 06 April 2015}

\section{References}

1. Birgand G, Blanckaert K, Carbonne A, Coignard B, Barbut F, Eckert C et al. Investigation of a large outbreak of Clostridium difficile PCR-ribotype 027 infections in northern France, 2006-2007 and associated clusters in 2008-2009. Euro Surveill. 2010;15(25).

2. Surveillance for community-associated Clostridium difficile-Connecticut 2006. MMWR Morb Mortal Wkly Rep. 2008;57(13):340-3.

3. Arvand M, Moser V, Schwehn C, Bettge-Weller G, Hensgens MP, Kuijper EJ. High prevalence of Clostridium difficile colonization among nursing home residents in Hesse, Germany. PLoS One. 2012;7(1):e30183.

4. Viseur N, Lambert M. Epidemiologie van Clostridium difficile infecties in België - Scientific Institute of Public Health, Belgium. WIV-ISP report 2012-22, (e-ISSN 2034-4635): 44.

5. Viseur N, Lambert M, Delmee M, Van Broeck J, Catry B. Nosocomial and non-nosocomial Clostridium difficile infections in hospitalised patients in Belgium: compulsory surveillance data from 2008 to 2010. Euro Surveill. 2011;16(43)

6. Miyajima F, Roberts $P$, Swale A, Price $V$, Jones $M$, Horan $M$ et al. Characterisation and carriage ratio of Clostridium difficile strains isolated from a community-dwelling elderly population in the United Kingdom. PLoS One. 2011;6(8).

7. Muyldermans G, Litzroth A, Ducoffre G, Quoilin S. Establishment and reinforcement of the national reference centers for human microbiology in Belgium. Arch Public Health. 2012;70(1):16.

8. Barbut F, Mario N, Delmee M, Gozian J, Petit JC. Genomic fingerprinting of Clostridium difficile isolates by using a random amplified polymorphic DNA (RAPD) assay. FEMS Microbiol Lett. 1993;114(2):161-6.

9. Drubbel I, de Wit NJ, Bleijenberg N, Eijkemans RJ, Schuurmans MJ, Numans ME. Prediction of adverse health outcomes in older people using a frailty index based on routine primary care data. J Gerontol A Biol Sci Med Sci. 2013;68(3):301-8.

10. Rambouskova J, Slavikova M, Krskova A, Prochazka B, Andel M, Dlouhy P. Nutritional Status Assessment of Institutionalized Elderly in Prague. Czech Republic Ann Nutr Metab. 2013;62(3):199-204.

11. Folstein MF, Folstein SE, McHugh PR. Mini-mental state. A practical method for grading the cognitive state of patients for the clinician. J Psychiatr Res. 1975;12(3):189-98.

12. Kuijper EJ, Barbut F, Brazier JS, Kleinkauf N, Eckmanns T, Lambert ML et al. Update of Clostridium difficile infection due to PCR ribotype 027 in Europe, 2008. Euro Surveill. 2008;13(31).
13. O'Connor JR, Johnson S, Gerding DN. Clostridium difficile infection caused by the epidemic BI/NAP1/027 strain. Gastroenterology. 2009;136(6):1913-24.

14. Kyne L, Merry C, O'Connell B, Kelly A, Keane C, O'Neill D. Factors associated with prolonged symptoms and severe disease due to Clostridium difficile. Age Ageing. 1999;28(2):107-13.

15. Mascart G, Delmee M, Van Broeck J, Cytryn E, Karmali R, Cherifi S. Impact of ribotype 027 on Clostridium difficile infection in a geriatric department. Eur J Clin Microbiol Infect Dis. 2013.

16. Goorhuis A, Debast SB, Dutilh JC, van Kinschot CM, Harmanus C, Cannegieter SC, et al. Type-specific risk factors and outcome in an outbreak with 2 different Clostridium difficile types simultaneously in 1 hospital. Clin Infect Dis. 2011;53(9):860-9.

17. Hensgens MP, Goorhuis A, Dekkers OM, van Benthem BH, Kuijper EJ. All-Cause and Disease-Specific Mortality in Hospitalized Patients With Clostridium difficile Infection: A Multicenter Cohort Study. Clin Infect Dis. 2013;56(8):1108-16.

\section{Submit your next manuscript to BioMed Central and take full advantage of:}

- Convenient online submission

- Thorough peer review

- No space constraints or color figure charges

- Immediate publication on acceptance

- Inclusion in PubMed, CAS, Scopus and Google Scholar

- Research which is freely available for redistribution

Submit your manuscript at www.biomedcentral.com/submit
C) Biomed Central 